\title{
Die Frage der sogenannten „Neuritis ascendens".
}

\section{Von}

\author{
Dr. G, C. Bolten (Haag, Holland).
}

Über die Möglichkeit des zentralwärts Aufsteigens neuritischer Prozesse ist viel und lange gestritten worden. Remak ${ }^{1}$ ) hat schon im Jahre 1861 die Aufmerksamkeit auf dieses Syndrom hingelenkt: „Ein Mann, der sich vor 3 Monaten das Nagelbett am kleinen Finger verletzt hatte, zeigt, obgleich sich die Wunde schon nach 14 tägiger Eiterung geschlossen hatte, Krampf und Lähmungserscheinungen an der Hand. Der Nervus ulnaris ist oberhalb des Epikondylus ganz deutlich geschwollen und druckempfindlich". Hier hatte also ein eitriger Entzündungsprozeß an der äußersten Peripherie sich bis weit höher hinauf fortgepflanzt und dabei aller Wahrscheinlichkeit nach den Lymphbahnen des Nerven gefolgt. Diesen Verlauf treffen wir in Wirklichkeit bei allen unter dem obenerwähnten Namen beschriebenen Fällen an. Nach Remaks Mitteilung vom Jahre 1861 bleibt die Neuritis ascendens in der Literatur längere Zeit ruhen. Später erscheinen wieder zahlreiche Vitteilungen über diesen Gegenstand. Remak jr. ${ }^{2}$ ), Krehl ${ }^{3}$ ), Oppenheim ${ }^{4}$ ), Lancereaux $x^{5}$ ), Kausch ${ }^{6}$ ), Marinesco $0^{7}$ ), beschrieben Fälle, bei welchen, unbedeutende Unterschiede untereinander nicht mitgerechnet, in der Hauptsache dieselbe Reihenfolge und dieselben Einzelheiten der Erscheinungen angetroffen werden. Es fängt immer mit einer Hautverletzung, welche infiziert ist, mit einem Abszeß oder mit

1) Nothnagels spez. Path. u. Ther. XI. Bd. 3 II, S. 92.

2) Remak u. Flatau, Neuritis u. Polyneuritis, Wien 1900, S. 265.

3) Lehrb. d. Nervenkrankh., Berlin 1913, Bd. I, S. 548.

4) Mitteil. a. d. Grenzgeb. der Med. u. Chir. Bd. I, S. 391.

5) M. de Fleury, Manuel pour l'étude des maladies du système nerveux, Paris 1904, S. 707.

6) Deutsche med. Wochenschr. 1896, S. 151 (Vereinsbeilage).

7) Deutsche med. Wochenschr. 1898, S. 57. 
einem anderen Entzïndungsherd an und ein ganz in der Nähe verlaufender Nervenstamm oder Hautnerv wird zu gleicher Zeit verwundet, wenigstens infiziert und nach einiger Zeit zeigt sich, daß dieser durch Bakterien hervorgerufene Prozeß, mehr oder weniger bedeutend zentralwärts in den Nervenstamm aufgestiegen ist. Daß dieses wirklich der Fall ist, wird von den genannten Autoren auf ganz genügenden Gründen mit zahlreichen klinischen Erscheinungen bewiesen. Auch in einer früher von mir $^{1}$ ) gemachten Mitteilung handelte es sich ausschließlich um infizierte Wunden, während in einem Fall bei einem tuberkulösen Manne sich infolge eines Traumas (jedoch ohne äußere Verletzung) dieselbe Eigentümlichkeit, nämlich das Aufsteigen eines infektiösen neuritischen Prozesses, vorkam. Es bleibt aber noch die wichtige Frage, ob in solchen Fällen, bei welchen nämlich Infektionskeime, welche in den Lymphbahnen eines Nervenstammes hineingeraten sind und sich in denselben zentralwärts auszubreiten und aufzusteigen wußten, von einer "Neuritis ascendens" die Rede sein kann. Es ist in der Tat sehr unwahrscheinlich, daß ein echter neuritischer Prozeß aufsteigen kann; wird die Verbindung zwischen dem peripheren Neuron und dessen Ganglienzelle zerstört, so degeneriert der peripher von der Läsion gelegene Teil in toto; wird, wie in den meisten Fällen von Neuritis und Polyneuritis geschieht, ein Nervenstamm durch endo- oder exogen-toxische oder dyskrasische Einflüsse zur Degeneration gebracht, dann tritt diese Entartung mehr oder weniger gleichzeitig über eine große Ausdehnung auf, ohne daß hierbei von einer Wanderung des Prozesses von der Peripherie zum Zentrum sich etwas zeigt. Bei weitem die meisten Fälle von Neuritis und Polyneuritis zeigen denn auch pathologisch-anatomisch keine spezifischen Entzündungserscheinungen, sondern ausschließlich Zeichen von Entartung. Der Achsenzylinder zerfällt zuerst und verliert seinen Zusammenhang; aus den Fibrillen entstehen unregelmäBige, perlenschnurartige Reste, welche ziemlich schnell resorbiert werden und auch die Markscheide fängt an zu degenerieren und in größere und kleinere zylindrische Stiucke auseinanderzufallen. Bei einem solchen Prozeß kann von einem Aufsteigen von der Peripherie zum Zentrum keine Rede sein. Es gibt jedoch noch andere neuritische Prozesse, bei welchen sowohl die Ätiologie wie der Verlauf ein wenig anders sind, und zwar die interstitiellen Neuritiden. Bei diesen findet sich ein, auch pathologisch-anatomisch, echter Ent- 
zündungsprozeß, die Gefäße des Nerven (welche im Endo- und Perineurium verlaufen) sind überfïllt, und es bildet sich ein Exsudat, welches das Bindegewebe und das Perineurium auseinanderdrängt. Eine ganze Menge von Leukocyten wandern in das Exsudat ein, und es bildet sich ein ausgebreitetes Infiltrat, entweder diffus oder mit mehreren lokalen Herden. Streifen- oder punktförmige Blutungen treten am ganzen Stamm auf; gewöhnlich schwindet nach einiger Zeit die Hyperämie; es kann bisweilen zur Eiterbildung kommen. Das Ende ist fast immer eine mehr oder weniger starke Bindegewebswucherung, welche infolge der Schrumpfung zu starken Ernährungsstörungen des Achsenzylinders führt und schließlich dessen totale Entartung bewirkt. Und ein solcher Prozeß, der also weit mehr ein echter Entzïndungsprozeß ist als die toxämischen oder dyskrasischen Neuritiden, kann entschieden aszendieren. Wenn in einer infizierten Wunde die Bakterien in die Lymphbahnen, welche sehr reichlich im Endo- und Perineurium vorhanden sind, eindringen, können sie sich sehr leicht und relativ schnell mit dem Lymphstrom zentralwärts bewegen und in dieser Weise zu einem aszendierenden Prozeß Anlaß geben. v. Strümpell11), der von einer echten "Neuritis ascendens" nichts wissen will, gibt denn auch zu, daß in seltenen Fällen echte Entzündungsprozesse in einem Nerv auf naheliegende Stämme übergreifen können. Wertheim Salomonson ${ }^{2}$ ) behandelt in seinem vortrefflichen Lehrbuch an mehreren Stellen die Neuritis ascendens, gibt aber dabei Erklärungen über das Wesen der Sache und Auffassungen, welche nicht ganz miteinander im Einklang sind. Erstens wird u. a. gesagt: „Die Entzündung kann sich ausbreiten in auf- und absteigender Richtung, was zur Aufstellung der Begriffe der Neuritis ascendens und descendens geführt hat. Weil die Erscheinungen des deszendierenden Neuritis klinisch nicht darzutun sind, redet man nur von einer Neuritis ascendens. Es hat sich aber gezeigt, daß, wenigstens klinisch, eine echte Neuritis ascendens zu den größten Seltenheiten gehört, seitdem man besser gelernt hat die hysterischen Paresen, die ischämischen und infektiösen Myositiden und die nicht aszendierenden Neuritiden davon abzutrennen. Es dünkt mich außerdem sehr wahrscheinlich, daB ein Teil der Fälle, welche wir klinisch als aszendierende Neuritis auffassen mïssen, und welche immer von infi-

1) Spez. Path. u. Ther. der inn. Krankh., Leipzig 1907, Bd. Ir, S. 385.

2) Path. e. Ther. der Neuritis, Myositis, Zenuwgezwellen, Neuralgie en Myalgie, Amsterdam 1911, S. 15, 24 u. 140. 
zierten Wunden herrühren, tatsächlich von einer langsam aufwärts schreitenden Lymphangoitis, welche auf den Nervenstamm übergreift, abhängig ist."

Aber weiter heißt es: „Bei offenen Wunden an den distalen Enden der Extremitäten, wobei also die Gelegenheit zur Infektion geboten wird, entwickelt sich nicht selten eine lokale Neuritis. In Ausnahmefällen kann diese einen aszendierenden Charakter annehmen und auch von einem Nerv auf einen andern übergreifen. Bei der Neuritis ascendens kann an ein Fortschreiten der Entzündung, das perineurale Gewebe entlang, gedacht werden." Diese Erklärung ist m. E. die einzig richtige, wie ich nachher noch zu bestätigen hoffe. Bei der Besprechung der traumatischen Neuritis ascendens sagt Wertheim Salomonson. aber erst, nachdem er den Fall Marinescos mitgeteilt hat (Infektion, von einer obliterierten Arterie bei Fußgangrän ausgehend; nachher entwickelt sich eine anatomisch festgestellte Neuritis mit Parese, Muskelatrophie, Anästhesien des Ober-und Unterschenkels usw.; hierbei wurde von Marinesco eine Streptokokkeninvasion im ganzen Verlauf des Nerven gefunden): ,während es à priori schon unwahrscheinlich ist, nach allem, was über die pathologischen Prozesse der peripheren Nerven bekannt ist, daß in einem Nervenstamm selber eine Entzündung fortgeleitet wird, zeigt es sich wohl, daß bei den meisten mitgeteilten Fällen der Beweis für die aufsteigende Neuritis fehlt. Im Gegenteil finden wir jedesmal Andeutungen, daß die Nervenentzündung entweder von einer Phlegmone oder von einer in der Nähe gelegenen Lymphangoitis und Adenitis abhängig ist. Letztere aszendiert und veranlaßt die neuritischen Erscheinungen. Diese Fälle mïssen also zu den septischen Neuritiden, oder besser, zu den Neuritiden infolge fortgeleiteter Entzündung gerechnet werden. " Es ist sehr wohl möglich, daß Wertheim Salomonson recht hat, und dab einige Fälle in der Literatur mit Unrecht als Neuritis ascendens beschrieben worden sind und in Wirklichkeit auf einer Kontiguitätsneuritis oder auf einer Lymphangoitis mit sekundären neuritischen Erscheinungen beruhen; aber dadurch fällt die Möglichkeit, welche auch von Wertheim Salomonson eingeräumt wird, absolut nicht fort, nämlich, daß ein Nervenstamm von einer eiternden Hautwunde aus infiziert wird, daß also Keime in die perineuralen Lymphbahnen eindringen, sich dann mit dem Lymphstrom zentralwärts bewegen und also ein Aufsteigen, ein Verlegen und Ausbreiten des perineuritischen Prozesses in zentripetaler Richtung zustande bringen können. In dieser Hinsicht ist der Fall Marinescos 
am deutlichsten $\mathbf{1}$ ); klinisch waren alle Erscheinungen einer immer höher hinaufsteigenden Neuritis vorhanden und bei der Autopsie wurde festgestellt, daß der Nerv ganz in dem gangränösen Herd am Fuße eingebettet war, weiter eine Streptokokkeninvasion im ganzen Nerven und selbst deutliche Veränderungen im Rückenmark (aber keine Streptokokken), Leukocyten in den Gefäßen der grauen Substanz und Strukturveränderungen in den Ganglienzellen. Marinesco nimmt an, daß diese mikoskopischen Veränderungen im Mark auf Vergiftungsprozessen beruhen, hervorgerufen durch Toxine, welche vermittelst der Lymphbahnen das Mark erreichten. Auch bei den zahlreichen und sehr ausführlichen Tierversuchen von Homan und Laitinin²) zeigte sich, daß Streptokokkenkulturen, bei Kaninchen im Nervus ischiadicus injiziert, sich im Nerven, den Lymphbahnen und großen Lymphräumen folgend, weiter zu verbreiten verstanden und zu wiederholten Malen das Rückenmark erreichten und sich dabei meistens in den hinteren Wurzeln anhäuften. Freilich gibt es keine Tatsachen oder Beobachtungen, welche die Möglichkeit ausschließen, daß Infektionskeime, welche an der Peripherie in den Lymphbahnen eines Nerven hineingeraten sind, darin zentralwärts aufsteigen. Ich meine denn auch einen Fall beobachtet zu haben, in dem ein solcher perineuritischer, infektiöser Prozeß, der klinisch alle Erscheinungen der Neuritis ascendens darbot, im Spiele war:

N., 25 Jahre, Mechaniker bei der Telegraphie; blasser Mann, der seine Arbeit immer gut hat verrichten können, bekommt auf einmal ziemlich heftige Schmerzen in der rechten Hand, in der Nähe des Handgelenks, und, wie ex meint, infolge einer ziemlich großen Anstrengung der Hand, beim Heben eines schweren Hammers. Er arbeitete zwar weiter, aber die Schmerzen blieben bestehen und er meinte auch, die Kraft der Hand würde geringer. Der Hausarzt stellte Atrophie der Daumenballenmuskeln fest; später sah ich den Kranken. Er zeigt das Bild einer leichten Medianuslähmung und vielleicht auch eine leichte Schwellung in der Nähe des Os lunatum. Diese Schwellung ist ziemlich hart, ohne Verfärbung der Haut, ohne Fluktuation und ohne deutliche Veränderungen am Knochen; auch X-Photos zeigen nichts Positives. Wassermann ist negativ. Pirquet mäßig stark positiv. Inzwischen nehmen die Erscheinungen allmählich zu, die Schwellung wird deutlich sichtbar und die Druckempfindlichkeit an dieser Stelle nimmt zu, wie die Atrophie am Thenar und auch treten leichte Sensibilitätsstörungen auf; auch fängt der Kranke an über fortwährend heftiger werdende Schmer-

1) La Presse médicale, 23 Novembre 1898.

2) Beitr. z. path. Anatomie 1899, Bd.25, Nr. 1. 
zen am Vorderarm zu klagen. In der Tat entwickeln sich dort allmählich degenerative Muskelatrophien mit teilweiser Entartungsreaktion. Der Kranke ist während längerer Zeit poliklinisch behandelt worden (künstliche Sonne, Röntgenbestrahlung, Arsonvallisation), jedoch ohne irgendwelchen Erfolg. Er entzog sich der Behandlung und ließ sich anderwärts untersuchen, wo man an Syringomyelie dachte. Nach einigen Monaten sah ich den Kranken wieder; die Hand war an der Stelle des Os lunatum stark angeschwollen und es bestand zweifellos ein Tuberkuloseherd in dem Os lunatum, aber außerdem klagte er über sehr heftige brennende Schmerzen im ganzen rechten Arm und in der Schulter. Er zeigte damals das Bild einer vollständigen Plexusneuritis: degenerative Muskelatrophie (mit partieller Entartungsreaktion) aller Arm- und Schultermuskeln, hier und da hyperästhetische Stellen, sehr große Druckempfindlichkeit des Plexus brachialis und der Nervenstämme. Der Nervus ulnaris ist leicht zu fühlen über dem Epicondylus int., der Nervenstamm ist verdickt und sehr druckempfindlich. Die Muskeln der Hand sind alle sehr atrophisch und Bewegungen des Daumens und der Finger sind völlig unmöglich. Biegungen im Ellenbogengelenk sind noch ein wenig möglich. Erhebung des Armes kann nur in minimaler Weise stattfinden.

Er wird ins Krankenhaus aufgenommen; jede Behandlung (künstliche Sonne, Röntgenstrahlen) ist völlig erfolglos, und auf des Kranken wiederholte Bitte wird, der unerträglichen Schmerzen wegen, zur Amputation des Vorderarmes unter dem Ellenbogengelenk, beschlossen. Das Os lunatum zeigte sich ganz zerstört und aufgelöst in einem großen, tuberkulösen Herd, in welchem auch der Nervus med. ganz aufgenommen war; die großen Nervenstämme waren stark angeschwollen, ziemlich ödematös und sehr blutreich, so daß eine blutige Flüssigkeit sich sehr leicht von der Schnittfläche abstreichen ließ, und befanden sich in einem deutlichen Entzündungszustand. (Zu meinem Bedauern ist die mikroskopische Untersuchung unterblieben.) Nach Ablauf der Operation hat sich der Kranke schnell gebessert; zwar sind die Muskelatrophien unverändert bestehen geblieben, aber die Schmerzen sind allmählich ganz geschwunden, so daß er nach ein paar Wochen befriedigt das Krankenhaus verließ.

Dieser Fall ist m. E. vollständig klar: es besteht ein tuberkulöser Herd in einem Handwurzelknochen, dieser Entzïndungsprozeß verbreitet sich stark über die Weichteile und auch über den Nerv. med. und offenbar dringen Tuberkelbazillen in die Lymphbahnen des Nerven ein. Im Anfang zeigen sich deshalb nur die Erscheinungen der Neuritis, welche sich aber auf den meist peripheren Teil des Nerv. med. beschränkt. Erst einige Monate später deuten die Erscheinungen darauf hin, daß der Prozeß sich über den im Vorderarm verlaufenden Teil des Nerven ausgedehnt hat und erst wieder nach Verlauf mehrerer Monate zeigt der Kranke das Bild einer vollständigen Plexusneuritis. Mit Bestimmtheit kann ich mitteilen, daß, als der Kranke zum ersten 
Male zu mir kam, sein Vorderarm ganz normal war und keine neuritischen Symptome zeigte; auch fand sich nie eine Spur weder von Lymphangoitis noch von irgendwelcher Drüsenschwellung. Der Tuberkuloseprozeß ist denn auch immer auf das Os lunatum beschränkt geblieben und von dort aus sind die Lymphgefäße des Nerv. med. infiziert. Später - der ganze Prozeß hat mehr als ein Jahr gedauert - müssen auch die Lymphbahnen des Plexus brachialis infiziert worden sein, denn schließlich waren auch die Oberarm- und die Schultermuskeln stark atrophisch. Außer dem primären Knochenherd und der Neuritis interstitialis war bei diesem Kranken nirgendwo etwas wie Entzündung zu entdecken; die Lymphdrüsen waren gleichfalls an keiner Stelle geschwollen, so daB hier ganz gewiß nicht von einer aszendierenden Lymphangoitis mit sekundären Neuritiserscheinungen die Rede sein kann.

In ungefähr allen bis jetzt behandelten Fällen von Neuritis ascendens, welche der Kritik standhalten, handelt es sich immer um eine infizierte Hautwunde, eine Phlegmone oder einen anderen Entzïndungsherd und in diesem Herd werden die Lymphbahnen des Nervenstammes, welcher immer in einem mehr oder weniger innigem Kontakt mit diesem Herd steht, infiziert. Es sind aber auch Fälle beschrieben worden, wo diese Infektionsquelle fehlte; so teilt Lichtenberger ${ }^{1}$ ) den Fall eines Mannes mit, der nach einem Trauma am Fußrucken, aber ohne Hautverletzung, progressive Erscheinungen einer Tibialis- und Peroneuslähmung zu zeigen anfing (sehr starke Muskelatrophien, schwere Entartungsreaktion, Sensibilitätsstörungen). Vermutlich werden in solchen Fällen - ohne infizierte Hautwunde - wohl, wie Wertheim Salomonson mit Recht behauptet, die klinischen Erscheinungen auf andere Weise zu erklären sein.

Ubrigens herrscht auf diesem Gebiet noch ein großer Mangel an Übereinstimmung. So will Zalla ${ }^{2}$ ) unter dem Namen ',Neuritis ascendens" gerade jene Prozesse verstehen, bei welchen eine echte Neuritis im Spiele ist und dagegen die Fälle, bei welchen die peripheren Nerven durchwandert worden sind von Bakterien oder durchtränkt mit Toxinen, welche zentripetal aufsteigen, und bei denen die Nerven selbst nicht der Sitz eines neuritischen Prozesses geworden sind, ausschließen. Die Neuritis ascendens in diesem Sinne ist nach Zalla eine ziemlich

1) Referat Neurol. Zentralbl. 1913.

2) La Névrite ascendante, Florence 1913. 
seltene Erkrankungsform; ätiologisch kommen Traumata mit Infektion der offenen Wunde in Betracht; nur ausnahmsweise können auch nicht offene Verletzungen den Ausgangspunkt eines aszendierenden neuritischen Prozesses bilden. Unter den ätiologischen Faktoren hat vielleicht die Prädisposition $\mathrm{zu}$ chronischen Intoxikationen (Toxïnfektionen) eine gewisse Bedeutung. Zalla unterscheidet drei Phasen: die peripherische, die ganglionäre und die radikulo-medulläre Phase. Nach Zalla ist die erste konstant, die zweite aber nicht regelmäßig vorhanden; betreffs der dritte Phase ist es unsicher, ob sie klinisch schon beobachtet ist. Das Tierexperiment hat gezeigt, daß pathogene Keime oder ihre Toxine imstande sind, längs der peripheren Nerven bis zu den Spinalganglien und dem Rückenmark emporzusteigen, indem, sie längs ihres Weges eine deutliche Entzündung hervorbrachten; es ist aber nicht gelungen beim Tier eine Neuritis ascendens im Sinne Zallas zu erzeugen.

Zalla meint also, daß eine echte, parenchymatöse (nicht entziundliche) Neuritis aszendieren kann, und rechnet die infektiöse Perineuritis (oder Lymphangoitis des Nerven) nicht zur Neuritis ascendens.

Auch Lichtenberger meint in seinem oben erwähnten Fall, daß das Trauma die Nervensubstanz des Tibialis und Peroneus lädierte, und daß dadurch eine Nervendegeneration auftrat, welche sich zentripetal fortsetze. Lichtenberger glaubt also, wie auch Zalla, daß in diesen Fällen eine echte aszendierende Neuritis parenchymatosa im Spiele ist.

Diese Vorstellung scheint mir völlig unrichtig und macht die Sache undeutlich; eine echte Neuritis, ein parenchymatöser Prozeß, kann nicht aszendieren; die Lymphbahnentziundung hingegen (also die Perineuritis oder die interstitielle Neuritis) wohl, und dabei entstehen schließlich die sekundären Erscheinungen der Druckneuritis.

Ich meine denn auch, aus dem hier Beschriebenen folgern zu diurfen, daß eine echte Neuritis ascendens nicht besteht. Wohl aber können Entzündügsprozesse in den Lymphbahnen der Nerven sich in zentripetaler Richtung ausbreiten; um aber eine Begriffsverwirrung zu vermeiden, sollten wir in jenen Fällen eigentlich von einer „Lymphangoitis nervorum ascendens" reden und könnten dann noch hinzufügen „streptococcica", ,tuberculosa" usw. Solche Fälle sind in Wirklichkeit unzweifelhaft sehr selten; der hier mitgeteilte ist der einzige, welchen ich in den letzten zehn Jahren sah. 